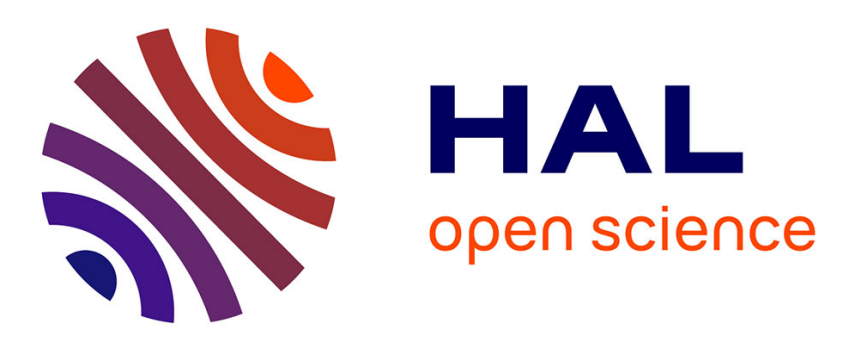

\title{
Paradoxical drying of a fired-clay brick due to salt crystallization
}

Sonia Gupta, Hendrik P. Huinink, Marc Prat, Leo Pel, Klass Kopinga

\section{To cite this version:}

Sonia Gupta, Hendrik P. Huinink, Marc Prat, Leo Pel, Klass Kopinga. Paradoxical drying of a firedclay brick due to salt crystallization. Chemical Engineering Science, 2014, vol. 109, pp.204-2011. 10.1016/j.ces.2014.01.023 . hal-00999299

\section{HAL Id: hal-00999299 https://hal.science/hal-00999299}

Submitted on 3 Jun 2014

HAL is a multi-disciplinary open access archive for the deposit and dissemination of scientific research documents, whether they are published or not. The documents may come from teaching and research institutions in France or abroad, or from public or private research centers.
L'archive ouverte pluridisciplinaire HAL, est destinée au dépôt et à la diffusion de documents scientifiques de niveau recherche, publiés ou non, émanant des établissements d'enseignement et de recherche français ou étrangers, des laboratoires publics ou privés. 


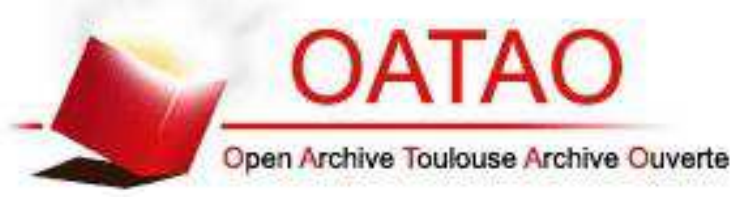

\section{Open Archive TOULOUSE Archive Ouverte (OATAO)}

OATAO is an open access repository that collects the work of Toulouse researchers and makes it freely available over the web where possible.

This is an author-deposited version published in : http://oatao.univ-toulouse.fr/ Eprints ID : 11731

To link to this article :

DOI:10.1016/j.ces.2014.01.023

URL : http://dx.doi.org/10.1016/j.ces.2014.01.023

\section{To cite this version :}

Gupta, Sonia and Huinink, Hendrik P. and Prat, Marc and Pel, Leo and Kopinga, Klass Paradoxical drying due to salt crystallization. (2014) Chemical Engineering Science, vol. 109 . pp. 204-2011. ISSN 0009-2509

Any correspondance concerning this service should be sent to the repository administrator: staff-oatao@ listes-diff.inp-toulouse.fr 


\title{
Paradoxical drying of a fired-clay brick due to salt crystallization
}

\author{
Sonia Gupta ${ }^{a}$, Hendrik P. Huinink ${ }^{\mathrm{a}, *}$, Marc Prat ${ }^{\mathrm{b}, \mathrm{c}}$, Leo Pel ${ }^{\mathrm{a}}$, Klass Kopinga ${ }^{\mathrm{a}}$ \\ a Transport in Permeable Media, Department of Applied Physics, Eindhoven University of Technology, PO Box 513, 5600 MB Eindhoven, The Netherlands \\ ${ }^{\mathrm{b}}$ INPT, UPS, IMFT (Institute de Mecanique des Fluides de Toulouse), University de Toulouse - Allee Camille Soula, F-31400 Toulouse, France \\ ${ }^{\text {c } C N R S, ~ I M F T, ~ F-31400 ~ T o u l o u s e, ~ F r a n c e ~}$
}

\section{H I G H L I G H T S}

- Influence of crystallization on the formation of receding drying front.

- Critical moisture content is not an intrinsic property of a porous material.

- Critical moisture content varies depending on the initial drying rate.

- Initial drying rate influences efflorescence type formed at the material surface.

Keywords:

Drying

Porous media

Crystallization

Efflorescence

Salt weathering

Critical moisture content

\begin{abstract}
A B S T R A C T
We investigated how salt crystallization inside a porous building material influences the formation of a receding drying front. Nuclear Magnetic Resonance (NMR) is used to measure non-destructively both hydrogen and dissolved sodium ions during drying process. In this study, we focused on the influence of $\mathrm{NaCl}$ on the drying behavior of porous media. The results show that salt changes the drying behavior. At low relative humidities ( $\mathrm{RH} \sim 0 \%$ ), the drying rate of a brick saturated with $\mathrm{NaCl}$ solution is much lower than the drying rate of water saturated brick. Moreover, the presence of salt suppresses the development of a receding front. In this case homogenous drying of the material continues till very low saturation values. This is due to salt crystallization near the surface of the brick that causes blockage of the pores. This blockage reduces evaporation rate at the surface and allows maintaining a continuous hydraulic connection between the surface of the porous medium and the liquid present inside the material till low saturation values. In the case of a salt solution saturated brick, increasing relative humidity to $55 \%$ and $70 \%$ leads to a paradoxical situation where the evaporation rate is greater for $55 \%$ and $70 \% \mathrm{RH}$ than for $0 \% \mathrm{RH}$. The paradox is explained by the impact of evaporation rate on the efflorescence microstructure, leading to the formation of a blocking crust for sufficiently high evaporation rates and non-blocking efflorescence for sufficiently low evaporation rates. The fundamental difference between the two types of efflorescence is demonstrated from a simple imbibition experiment. Using a simple continuum scale model of drying, critical moisture content was determined and all the essential features of the experimental results are validated. It is shown that critical moisture content can be very low in the case of fired-clay brick due to the low threshold of the pore space, which is consistent with the relatively large pore size distribution of the fired-clay brick.
\end{abstract}

\section{Introduction}

Salt crystallization inside the pores of a building material can cause severe damage (Scherer, 1999) and is known since ancient times (Taber, 1916). Salt can enter a building material along with moisture in various ways, such as capillary rise of ground water, absorption of sea water, atmospheric pollution. Almost all water that enters building materials will leave by evaporation or drying.
Due to drying salt crystals will crystallize inside the pores of a building material.

Drying of porous media in the presence of salt is of interest not only to understand the crystallization mechanisms and related salt damage problems, but also in the field of soil physics (Nachshon et al., 2011), injecting $\mathrm{CO}_{2}$ in aquifers (Peysson et al., 2011), and related studies. Vegetation, plant growth and soil organisms can be severely limited in salt-affected land. Despite the above mentioned problems, drying of porous materials in the presence of salt is still poorly understood. This is due to the complex phenomena involved, e.g., moisture and ion transport phenomena and phase transitions. Therefore, a better insight into the drying process of 
salt containing porous media is required to understand various salt related damage problems.

Lewin (1982) proposed that the location of salt crystallization is determined by a dynamic balance between the rate of escape of water at the surface (evaporation or drying) and the rate of supply of water to the surface. If salt crystallizes inside the material, i.e., the socalled sub-florescence, it can cause damage. On the other hand, if salt crystallizes outside the material, also called efflorescence, it may be unesthetic but it is not harmful for the buildings. The location of salt crystallization is in general determined by the competition of diffusive and advective fluxes. Generally it is characterized in terms of a Peclet number (Pe) (Huinink et al., 2002).

In the past, a significant progress has been made in understanding the drying kinetics of porous materials, especially focusing on the pore level (Prat, 2007; Prat and Bouleux, 1999). It is widely mentioned that for irregular pore geometry thick liquid films provide an efficient transport of liquid to the surface (Chauvet et al., 2009). The pores inside the brick are generally not perfectly cylindrical and have edges; consequently thick liquid films will be present. These films are known to contribute significantly to the mass transport phenomenon during stage-1 of drying (Laurindo and Prat, 1998). The films supply water to isolated liquid clusters, thereby enhancing the mass transfer in comparison to the vapor diffusion transport mechanism (Laurindo and Prat, 1998). The standard drying behavior of water saturated porous material is well documented in the literature (Hall and Hoff, 2002). However, the drying behavior of the porous material in the presence of salt is still a topic of discussion, especially with regard to the impact of salt crystallization on drying. By varying the mean pore size of the porous material, it has been shown that the crystallization of the salt at the porous material surface could either severely reduce the evaporation rate compared to pure water or have no impact at all (Eloukabi et al., 2013). This led to identify two main types of efflorescence, referred to as patchy (or non-blocking) and crusty (or blocking). As we shall see, distinguishing these two main types of efflorescence is also a key factor for analyzing our results.

The aim of this work is to study the drying behavior of porous materials saturated with the solution. In this study we will focus on $\mathrm{NaCl}$ as this is a common salt found in many salt related problems in situ. We will investigate to which extent this drying behavior resembles the standard drying behavior of water saturated materials. In particular, we focus on the effect of salt crystallization inside a porous material on the development of a receding front during drying. To our best knowledge, no study has been reported in the past to explore this aspect of drying, probably because it is difficult to measure the moisture and ion distribution simultaneously in real porous materials. For this purpose a specially designed Nuclear Magnetic Resonance (NMR) set-up with a static magnetic field of $0.78 \mathrm{~T}$ was used (Kopinga and Pel, 1994). With this set-up it was possible to carry out non-destructive, quantitative and simultaneous measurements of both the hydrogen and sodium content in a brick sample.

\section{Material and methods}

\subsection{Materials}

The material used in this study was fired-clay brick. The red fired-clay brick is of a type typically used for construction in the Netherlands, and had an average porosity (as measured by water immersion method) of $0.32 \mathrm{~m}^{3} \mathrm{~m}^{-3}$, and a pore size distribution ranging from a few tens of nanometers to $100 \mu \mathrm{m}$ (with $80 \%$ of the total pore space corresponding to pores in the range $1-10 \mu \mathrm{m})$, as determined by Mercury Intrusion Porosimetry (MIP) (Gupta, 2013).

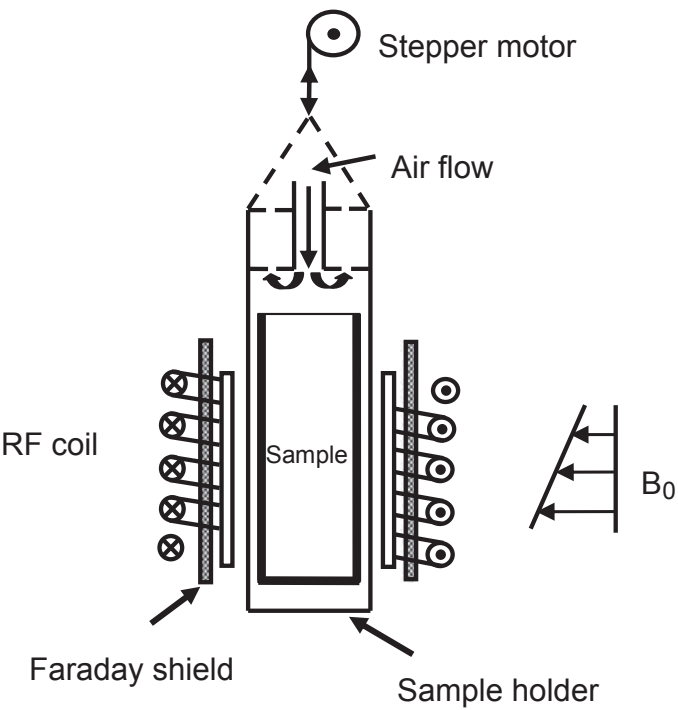

Fig. 1. NMR set-up for the drying experiments with cylindrical samples. The Teflon holder with the saturated sample is moved in the vertical direction by means of a stepper motor. The signal from $\mathrm{H}$ or $\mathrm{Na}$ nuclei at resonance is received by the RF coil.

\subsection{Nuclear magnetic resonance (NMR)}

A specially designed nuclear magnetic resonance (NMR) set-up, for non-destructive, quantitative and simultaneous measurement of moisture $\left({ }^{1} \mathrm{H}\right)$ and sodium $\left({ }^{23} \mathrm{Na}\right)$ profiles in inorganic materials was used. An extensive description of this set-up can be found elsewhere (Kopinga and Pel, 1994). A schematic representation of the set-up is given in Fig. 1. The tuned circuit of the set-up can be toggled between $33 \mathrm{MHz}$ for ${ }^{1} \mathrm{H}$ and $8.9 \mathrm{MHz}$ for ${ }^{23} \mathrm{Na}$, giving the possibility to quasi-simultaneously measure the $\mathrm{H}$ and $\mathrm{Na}$ content and thereby the concentration. For the echo time used in the experiment, i.e., $T_{E}=250 \mu \mathrm{s}$ for ${ }^{1} \mathrm{H}$ and $450 \mu \mathrm{s}$ for ${ }^{23} \mathrm{Na}$, only the dissolved $\mathrm{Na}$ and $\mathrm{H}$ nuclei are measured and no signal is obtained from the nuclei incorporated in the crystals. The magnetic field gradient was chosen so that a slice of less than $2 \mathrm{~mm}$ is measured. The cylindrical samples $20 \mathrm{~mm}$ in diameter and $40 \mathrm{~mm}$ in length were vacuum saturated with water and $3 \mathrm{~m} \mathrm{NaCl}$ solution. These samples were sealed using Teflon tape on all sides except the top surface and placed in the NMR sample chamber. In this way a one dimensional drying experiment was performed. The samples were then exposed to dry air at a flow rate of $11 \mathrm{~min}^{-1}$. The relative humidity was varied from $0 \%$ to $70 \%$. The sample was moved in the vertical direction using a stepper motor to allow the measurement of moisture and sodium content throughout the sample length. Measuring one profile takes about $2.26 \mathrm{~h}$. As the complete drying experiment takes in the order of a few days, small variations in the moisture and ion profiles during a single scan can be neglected. After each drying experiment was completed the efflorescence formed on the top of the sample was collected and weighed.

\section{Results}

\subsection{Drying behavior of water and salt solution saturated fired-clay brick dried at $0 \%$ relative humidity}

Initially, the drying behavior of fired-clay brick samples vacuum saturated with water and salt solution $(3 \mathrm{~m} \mathrm{NaCl})$ was studied. The samples were dried at $0 \% \mathrm{RH}$ and $11 \mathrm{~min}^{-1}$ air flow rate. Fig. 2a and b shows the measured moisture profiles during drying of water and salt saturated samples, respectively. The recurrent irregularities in the profiles result from inhomogenities 
a

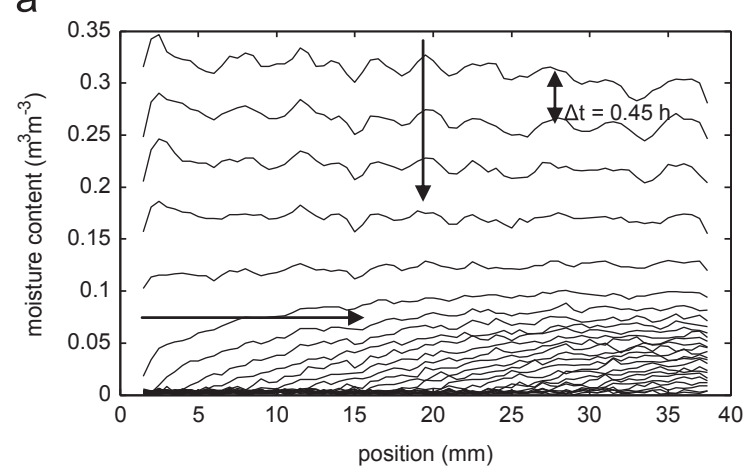

b

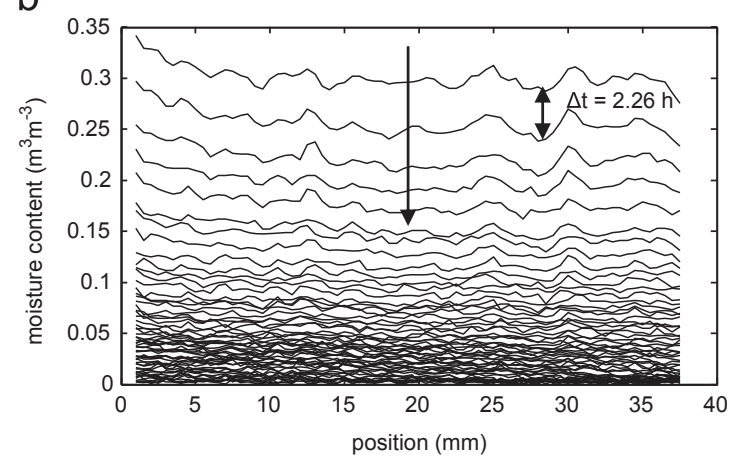

Fig. 2. The measured moisture profiles for (a) water saturated and (b) $3 \mathrm{~m} \mathrm{NaCl}$ saturated fired-clay brick plotted as a function of position. The profiles are given for every $0.45 \mathrm{~h}$ and $2.26 \mathrm{~h}$ for water and salt saturated bricks. The samples were dried using dry air with a flow of $11 \mathrm{~min}^{-1}$ and $0 \%$ relative humidity. The drying surface is at $0 \mathrm{~mm}$. The vertical arrow shows the homogenous drying of the sample (externally limited) and the horizontal arrow shows the penetration of the receding drying front (internally limited)

of the sample, e.g., at $\sim 15 \mathrm{~mm}$ in case of the water saturated sample (Fig. 2a).

In the case of water saturated sample, the first few profiles are almost horizontal, representing the first (externally limited) drying stage (shown by a vertical arrow in Fig. 2a). Afterwards, a drying front develops which recedes below the sample surface (shown by a horizontal arrow in Fig. 2a). This represents the second (internally limited) drying stage. The addition of salt changes the drying behavior of the fired-clay brick (Fig. 2b). Two effects were seen. First, the presence of $\mathrm{NaCl}$ reduces the drying rate compared to the drying rate of water saturated fired-clay brick. Complete drying of water saturated fired-clay brick took about one day, in comparison to more than one week for $\mathrm{NaCl}$ saturated fired-clay brick of the same dimensions and at the same drying conditions. Second, the receding drying front vanishes and homogenous drying of the material continues till low saturation values. Hence, drying stage- 1 is prolonged and homogenous drying is maintained till low saturation values.

\subsection{Drying behavior of water saturated fired-clay brick at high relative humidity}

Since the evaporation rates are markedly different between the pure water experiment and the salt experiment, it is interesting to get insights into the effect of evaporation rate on the drying process. To this end, experiments were performed on water saturated fired-clay brick at high humidity conditions, i.e., at $55 \%$ and $70 \% \mathrm{RH}$. Because of the depression of the humidity gradient at the surface, the drying rate should decrease. The evaporation rate is proportional to the difference between the relative humidity at the porous medium surface (which is equal to $100 \%$ when pure

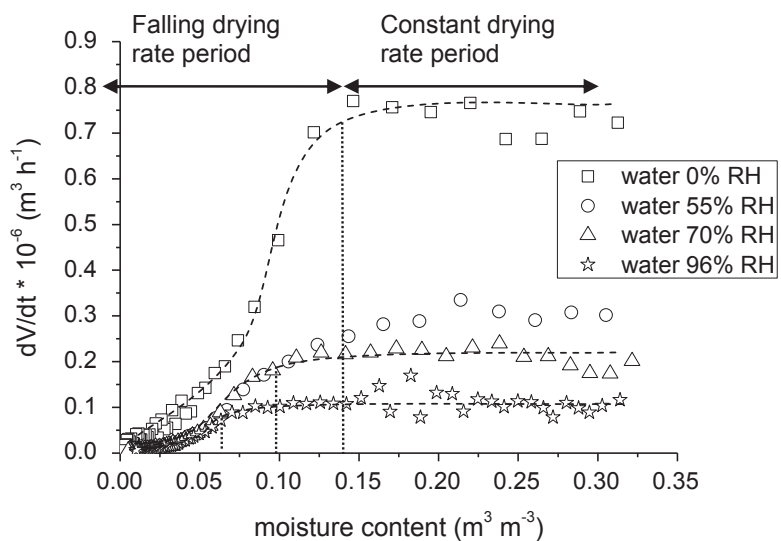

Fig. 3. The rate of water volume change $(d V / d t)$ as a function of moisture content $\left(\mathrm{m}^{3} \mathrm{~m}^{-3}\right)$ for water saturated fired-clay bricks dried at different relative humidity conditions. The dashed lines are guide to the eye.

liquid water is present at the surface and to $75 \%$ for a $\mathrm{NaCl}$ saturated solution) and the relative humidity in the external air.

The results of the drying experiments performed on water saturated samples are shown in Fig. 3. The rate of water volume change, i.e., $\mathrm{d} V / \mathrm{d} t$, is plotted as a function of moisture content. In this figure two drying stages can be distinguished, a constant drying rate period (stage-1) and a falling drying rate period (stage2 ). A constant drying rate corresponds to the externally limited drying stage and a falling drying rate period corresponds to the internally limited drying period. The dashed lines in Fig. 3 are drawn as a guide to the eye. The dotted vertical line denotes the moisture content at which the front starts to penetrate the sample. It can be clearly seen that at higher humidities the receding front starts to penetrate at lower moisture content values. The moisture content marking the end of stage- 1 is classically referred to as the "critical" moisture content (Hall and Hoff, 2002). For different materials the critical moisture content must be determined experimentally (Mujumdar, 1995) under a given set of environmental conditions. As can be seen from Fig. 3 the critical moisture content is in fact not constant but decreases with a decreasing evaporation rate.

In another experiment, a water saturated fired-clay brick was dried at $96 \%$ relative humidity while approx. $75 \%$ of the brick surface was covered with Teflon tape. Covering the brick surface was done to avoid evaporation and to mimic the situation of salt contaminated materials where the surface is blocked due to salt crystallization. These results are also plotted in Fig. 3. A significant delay (low saturation value) in the penetration of the receding front is seen. These results clearly confirm that the drying rate at the surface affects the penetration of receding front and the critical moisture content.

\subsection{Drying behavior of salt solution saturated fired-clay brick at high relative humidity}

An important result of the experiments described in the previous sub-section is that the evaporation rate at the surface of the material affects the formation of a drying front. In a separate study (Gupta, 2013) it was found that the evaporation rate of a salt contaminated brick was higher at high relative humidity than at low relative humidity. To investigate this, drying experiments were performed on samples saturated with salt solution at $55 \%$ and $70 \%$ relative humidity. The samples were vacuum saturated with $3 \mathrm{~m} \mathrm{NaCl}$ solution and dried inside NMR at room temperature and $11 \mathrm{~min}^{-1}$ air flow rate. The results are shown in Fig. 4, where the rate of water volume change $(\mathrm{d} V / \mathrm{d} t)$ is plotted as a function of moisture content. 
For comparison, the result of solution saturated brick dried at $0 \% \mathrm{RH}$ is also shown. For the brick dried at $0 \% \mathrm{RH}$, a continuous decrease of the flux with decreasing moisture content is seen. Thus, there is no constant rate period in this case. However, at high humidities initially a constant drying rate is maintained (stage-1) and later a falling drying rate period is observed (stage-2). This leads to a paradoxical drying situation since the evaporation rate is greater for $55 \% \mathrm{RH}$ and $70 \% \mathrm{RH}$ than for $0 \% \mathrm{RH}$. Thus, in the presence of $\mathrm{NaCl}$ a receding front develops again at high humidities. At the end of the experiment, the efflorescence formed on the surface of the fired-clay brick was collected and weighed. At $0 \%$ relative humidity $6-7 \%$ of the $\mathrm{NaCl}$ crystallized as efflorescence. This efflorescence had the form of a very thin crust on the outer surface of the fired-clay brick that strongly adhered to the substrate. It was not easy to remove the efflorescence from the substrate. Remaining salt was crystallized inside the brick as subflorescence. On the other hand, at 55\% and 70\% RH, a significant amount of $\mathrm{NaCl}$ crystallized as efflorescence. About $48 \%$ and $40 \%$ of the salt crystallized as efflorescence at $55 \%$ and $70 \% \mathrm{RH}$, respectively. The type of efflorescence formed at high humidities was rather fragile and was easy to remove from the substrate by rubbing. Pictures of the efflorescence formed on the surface of the materials are shown in Fig. 5. The efflorescence is clearly quite different at $0 \% \mathrm{RH}$ compared to the efflorescence at 55\% and 70\% RH. Similarly as in Eloukabi et al. (2013), this suggests distinguishing two types of efflorescence, referred to as "patchy" and "crusty" in Eloukabi et al. (2013) and that can be referred as well as "nonblocking" and "blocking", respectively. The efflorescence obtained at $0 \% \mathrm{RH}$ is blocking whereas the efflorescence at $55 \%$ and $70 \% \mathrm{RH}$ is non-blocking.

The blocking efflorescence does not form all of a sudden, but progressively covers the porous medium surface. This is explained by the non-uniformity of the evaporation flux at the porous

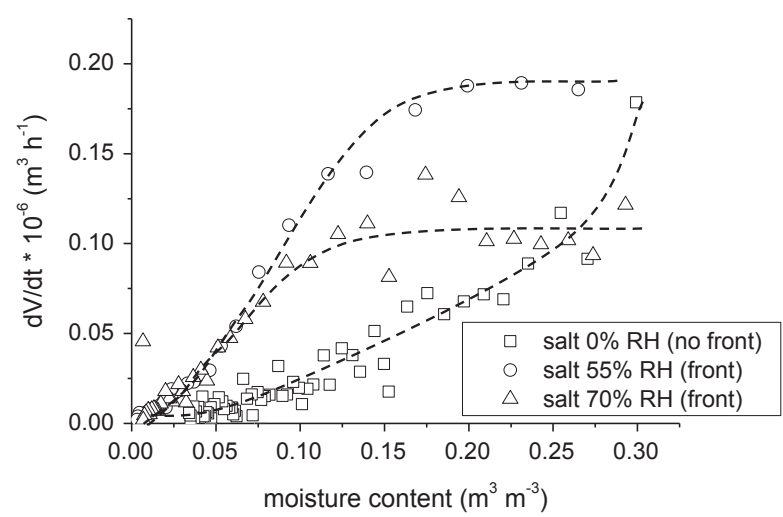

Fig. 4. The rate of water volume change $(d V / d t)$ as a function of moisture content $\left(\mathrm{m}^{3} \mathrm{~m}^{-3}\right)$ for salt saturated bricks dried at different relative humidity conditions. The dashed lines are a guide to the eye. medium surface. As explained for instance in Eloukabi et al. (2013) and Veran-Tissoires et al. (2012a), efflorescence starts forming in the region of higher evaporation fluxes at the surface and then progressively colonizes the rest of the surface. Other factors, such as possible local spatial variations of porosity and/or permeability, especially in the top region of the sample, may also play a role in the localization and development of efflorescence (Veran-Tissoires et al., 2012b). Thus, the pore blockage essentially starts where the evaporation flux is the highest and next progressively develops over rest of the surface of the material. The net effect is a progressive reduction of the surface left free for evaporation and consequently a continuous decrease of the flux with decreasing moisture content. As can be seen in Fig. 4, at low moisture contents $\left(0.15 \mathrm{~m}^{3} \mathrm{~m}^{-3}\right)$, the rate of water volume change $(\mathrm{d} V / \mathrm{d} t)$ is very low compared to the solution saturated fired-clay bricks dried at high humidities. However, at high humidities $\mathrm{NaCl}$ also crystallizes as efflorescence. Because of the formation of efflorescence we might expect a reduced blockage effect near the drying surface. Since we did not see any drop in drying rate after the formation of efflorescence, we conclude that the efflorescence is not transport limiting. Therefore, the system remains open and a constant volume flux is maintained initially, followed by a receding front. Thus, in this case, the efflorescence is nonblocking. An important outcome of the results shown in Fig. 4 is therefore that not only the mean pore size of the porous medium as in Eloukabi et al. (2013) but also the initial drying rate has an impact on the type of efflorescence that forms at the porous medium surface. This is qualitatively consistent with the crude efflorescence growth model introduced in Eloukabi et al. (2013), which "explains" the transition from non-blocking to blocking efflorescence from the consideration of viscous pressure drop into the growing efflorescence. Increasing the evaporation rate increases the pressure drop and thus the maximum height of efflorescence which then expands laterally rather than upwardly for a sufficiently high evaporation rate. The markedly different properties of blocking and non-blocking efflorescence are confirmed from the imbibition experiment described in the next subsection.

\subsection{Sorptivity test}

To estimate quantitatively the difference in pore blockage, bricks were dried at different humidity conditions and sorptivity tests were performed. The bottom of the test material is touched with decane to let the material absorb decane by capillary suction. The mass change is recorded as a function of time. The tests were performed on bare fired-clay brick and on fired-clay bricks saturated with $3 \mathrm{~m} \mathrm{NaCl}$ solution and dried at $0 \%$ and 55\% relative humidity. The main idea is that salt crystallization inside the pores causes a reduction of the pore volume and hence the permeability of the material, making solution transport more difficult

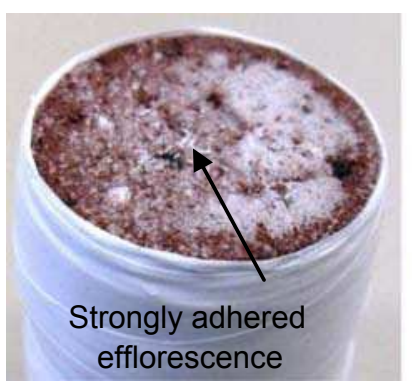

$0 \%$

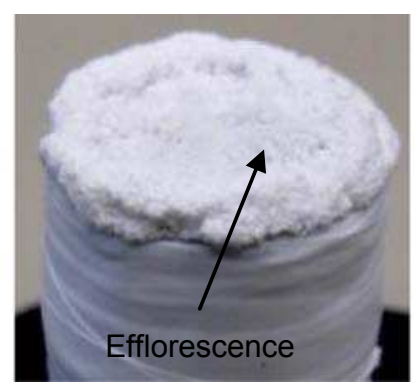

$55 \%$

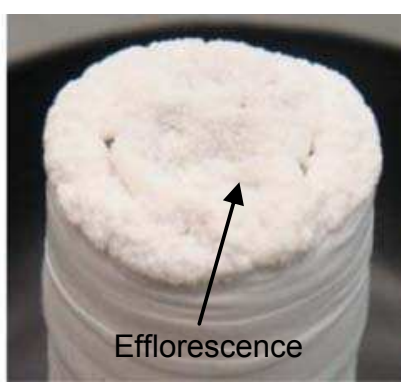

$70 \%$

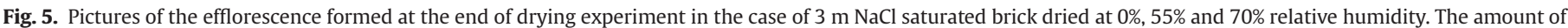
efflorescence increases at higher humidities. 


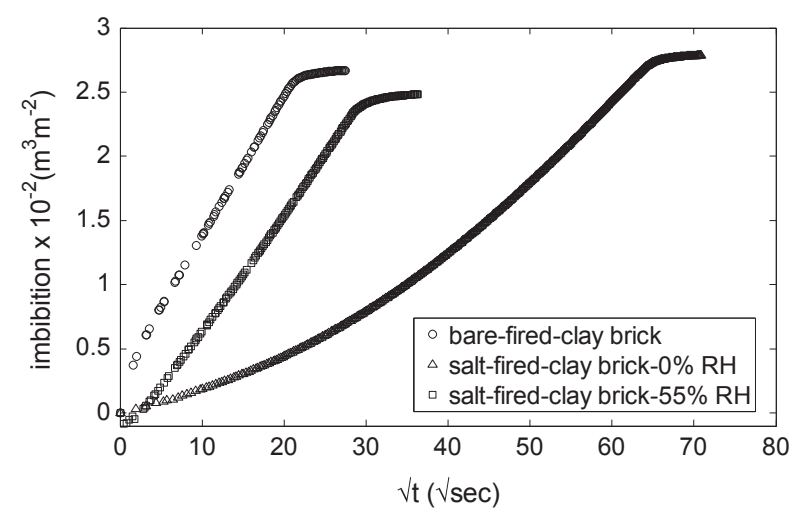

Fig. 6. The measured imbibition of decane in the salt-free fired-clay brick and in firedclay bricks saturated with $3 \mathrm{~m} \mathrm{NaCl}$ solution dried at different relative humidity conditions.

(Espinosa-Marzal and Scherer, 2013). Decane was used for sorptivity tests, because $\mathrm{NaCl}$ crystals do not dissolve in decane. We expect that blockage due to salt crystallization near the drying surface will generate a surface resistance to decane uptake in case of salt contaminated brick dried at $0 \% \mathrm{RH}$. Therefore, a delay in imbibition is expected. In the tests the efflorescence was not removed from the surface of the brick. This was done to check the permeability of the efflorescence also. The surface of the brick above which dry air was blown during the drying experiment was exposed to decane. The results for salt free, salt contaminated brick dried at $0 \% \mathrm{RH}$, and salt contaminated brick dried at $55 \%$ RH are shown in Fig. 6, where the imbibition $(i)$ is plotted as a function of the square root of time $(\sqrt{ } t)$. The imbibition is defined as the cumulative volume of absorbed decane per unit area of inflow surface. For the salt contaminated brick dried at $0 \% \mathrm{RH}$, the imbibition initially increases very slowly (slope of the curve is almost flat), indicating resistance to the uptake of decane. This is due to the presence of a dense layer of salt crystals near the drying surface that causes blockage. In depth of the sample the amount of crystallized salt and hence, the resistance to uptake of decane also decreases. Thus, the slope of the curve increases progressively. In case of the fired-clay brick dried at 55\% RH, no change in the slope of imbibition is observed, indicating that the permeability of the efflorescence does not limit the rate of fluid uptake.

\section{Discussion}

As presented in this section, the most important features of the experiments can be understood from a simple continuum scale model of drying.

\subsection{Water saturated brick}

To understand the different drying behaviors of $\mathrm{NaCl}$ saturated fired-clay brick we first have to understand the standard drying behavior of water saturated fired-clay brick. It is well understood that drying is a drainage process, where the non-wetting fluid (gas) replaces the wetting fluid (liquid). In general, drying of a homogeneous, uniformly wet, porous material has two identifiable stages, a uniform drying period (stage-1) followed by a receding drying front period (stage-2) as shown in Fig. 2a.

During stage-1, there is a continuous capillary flow of water towards the drying surface to meet the demands of evaporative flux (Hall and Hoff, 2002). Consequently, the rate of moisture loss from the surface of material is constant as long as the rate of liquid water that can be transported to the surface through the action of capillary forces is equal to the external evaporation demand. With time the unsaturated capillary flow can no longer supply water towards the drying surface at a rate high enough to meet the demands of the evaporative flux. This very classical scenario can be quantified using traditional continuum scale model. A key factor is to determine the "critical" moisture content marking the end of first drying period and thus the beginning of the formation of drying front within the porous medium (Fig. 2). During stage-1, the fact that the water brought to the surface thanks to capillary effects balances the evaporation demand which can be expressed as

$A K_{s}(\theta) \frac{\partial h}{\partial z}=-J$

where $A$ is the top surface area of the porous medium, $K_{s}(\theta)$ is the hydraulic conductivity of the porous medium, $h$ is the pressure head ( $h=P_{c} / \rho_{\ell} g$, where $P_{c}$ is the capillary pressure, $\rho_{\ell}$ is the density of liquid and $g$ is the acceleration due to gravity), $z$ is a vertical coordinate increasing downward from the top surface of porous medium, $J$ is the evaporation rate expressed in $\mathrm{m} / \mathrm{s}$. For the capillary regime considered in the present study, which is characterized by a spatially uniform moisture content during the first stage of drying, a first order approximation assuming $P_{c}$ varies linearly over $H$, the "critical" moisture content can be estimated from Eq. (1) expressing $\partial h / \partial z$ as $h(\theta) / H$, where $H$ is the height of sample. This gives

$A K_{S}(\theta) \frac{h(\theta)}{H}=-J$

To make use of Eq. (2), it is necessary to characterize the unsaturated hydraulic conductivity $K_{s}(\theta)$ and the retention curve $h(\theta)$. Following Carmeliet and Roels (2002), the moisture retention curve can be analytically described by a bimodal function of the van Genuchten (1980) type:

$\theta=\theta_{s}\left[\ell_{1}\left(1+\left(a_{1} p_{c}\right)^{n_{1}}\right)^{-m_{1}}+\ell_{2}\left(1+\left(a_{2} p_{c}\right)^{n_{2}}\right)^{-m_{2}}\right]$

As can be seen from Fig. 7, Eq. (3) with $\theta_{s} \approx 0.32, a_{1}=5.8 \times$ $10^{-5} \mathrm{~Pa}^{-1}, a_{2}=9 \times 10^{-6} \mathrm{~Pa}^{-1}, \ell_{1}=0.846, \ell_{2}=0.154, n_{1}=4, n_{2}=$ 1.69 and $m_{i}=1-n_{i}$ leads to a representation of the retention in good agreement with the few experimental data reported in Pel et al. (2010).

The unsaturated hydraulic conductance $K_{s}(\theta)$ is estimated from the retention curve using the Mualem (1976) model:

$K_{s}(\theta)=K_{s}\left(\theta_{s}\right)\left(\frac{\theta}{\theta_{c}}\right)^{0.5} \int_{0}^{\theta} \frac{d \theta}{p_{c}(\theta)} / \int_{0}^{\theta_{s}} \frac{d \theta}{p_{c}(\theta)}$

The saturated hydraulic conductance $K_{s}\left(\theta_{s}\right)$ can be evaluated from the fired-clay brick permeability $\kappa\left(\kappa \approx 9.6 \times 10^{-14} \mathrm{~m}^{2}, K_{s}\left(\theta_{s}\right)=\right.$ $\left.\kappa \rho_{\ell} g / \mu\right)$. Using the capillary pressure curve for fired-clay brick as given in Fig. 7 the relative permeability $k_{r}(\theta)=K_{s}(\theta) / K_{S}\left(\theta_{s}\right)$ can be calculated using Eq. (4) and is shown insert in Fig. 7. The calculated "critical" moisture content as a function of evaporation rate is obtained by inserting Eqs. (3) and (4) into Eq. (2) and solving for $\theta$. The result is shown in Fig. 8. Note that the intrinsic permeability $\kappa$ has been a bit modified $\left(\kappa=0.6 \times 10^{-14} \mathrm{~m}^{2}\right)$ so as to obtain critical moisture contents close to the ones obtained in the experiments (Fig. 3). This represents a reduction of the permeability by a factor 10 , which seems to be a quite acceptable change owing to the variability of permeability between different samples. As shown in Fig. 8, the "critical" moisture content decreases with a decreasing evaporation rate. The theoretical lower bound $\theta_{r}$ to the critical water content corresponds to the percolation threshold of the pore space pore network (Le Bray and Prat, 1999) and can be reached for an extremely low drying rate. The retention curve shown in Fig. 7 suggests that $\theta_{r}$ is very small for the fired-clay brick. It is order or smaller than the smallest moisture content 


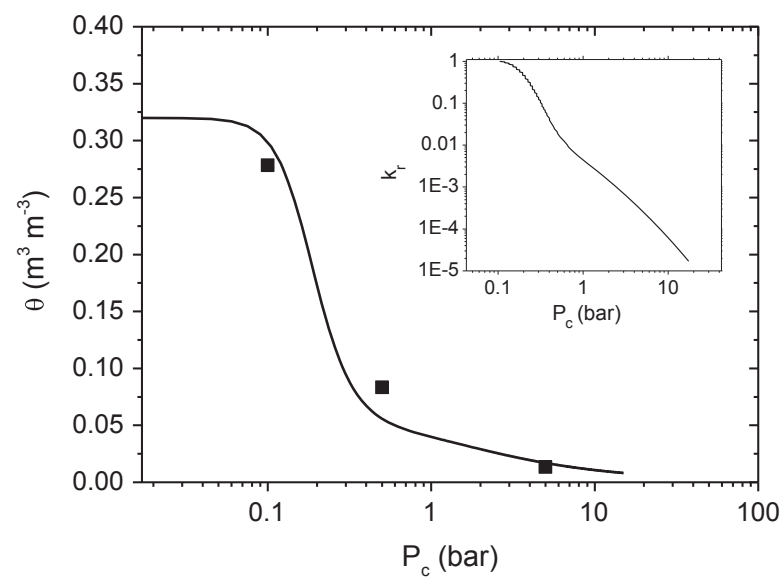

Fig. 7. Capillary pressure curve for fired-clay brick material. The filled symbols are experimental data (Pel et al., 2010). The solid line is computed using Eq. (3). The insert shows the relative permeability computed using Mualem's model (Eq. (4)).

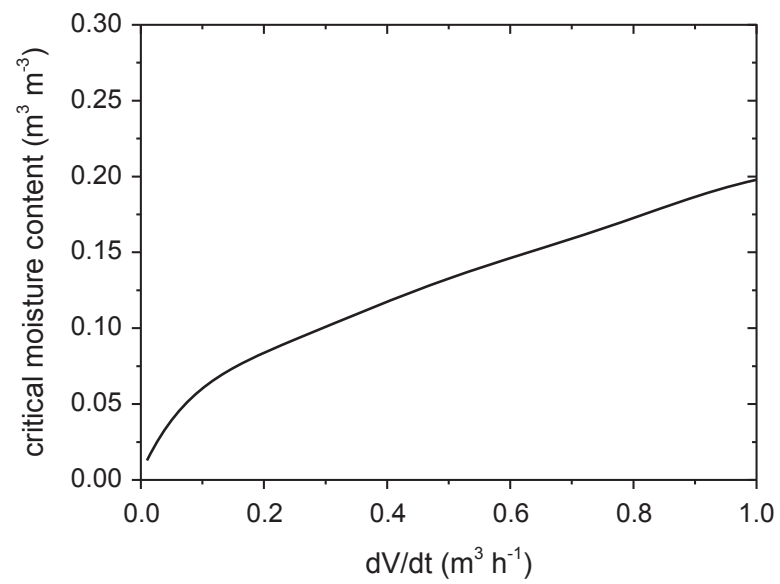

Fig. 8. Critical moisture content as a function of evaporation rate as predicted by Eq. (2).

reported in Fig. 2. This is consistent with experimental results at $0 \% \mathrm{RH}$ with the salt solution depicted in Fig. 2.

\subsection{Salt saturated brick}

Since the evaporation rate is very low in the presence of $\mathrm{NaCl}$ due to the formation of a blocking crust $(0 \% \mathrm{RH})$, the homogeneous drying can thus take place over most of the drying process in this case.

However, it can be pointed out that the evaporation rate is not constant, but decreases monotonously during stage-1 (=homogeneous drying) for the fired-clay brick dried at $\mathrm{RH}=0 \%$ (see Fig. 4). Hence Eq. (2) is thus slightly modified, to express explicitly the time dependence of evaporation rate in this case:

$A K_{s}(\theta) \frac{h(\theta)}{H}=-J(t)$

Here, we suppose that the salt crystallizes only within a thin layer at the top surface of porous sample. This is supported by the estimate of Peclet number $P e \approx J H / A D_{s}^{*}$, whereas $D_{s}^{*}$ is the effective diffusion of the ion in the porous medium $\left(D_{s}^{*} \approx 10^{-9} \mathrm{~m}^{2} / \mathrm{s}\right)$. For $J / \rho_{\ell}=0.1 \mathrm{~cm}^{3} / \mathrm{h}$, which is a mean representative value for the case of $0 \% \mathrm{RH}$ (see Fig. 4) this yields $P e \approx 3.5$. The Peclet number is greater than 1 and thus the accumulation of the salt at and near the top surface of sample is expected (Hall and Hoff, 2002). Salt crystallization near the top of the sample has also been confirmed

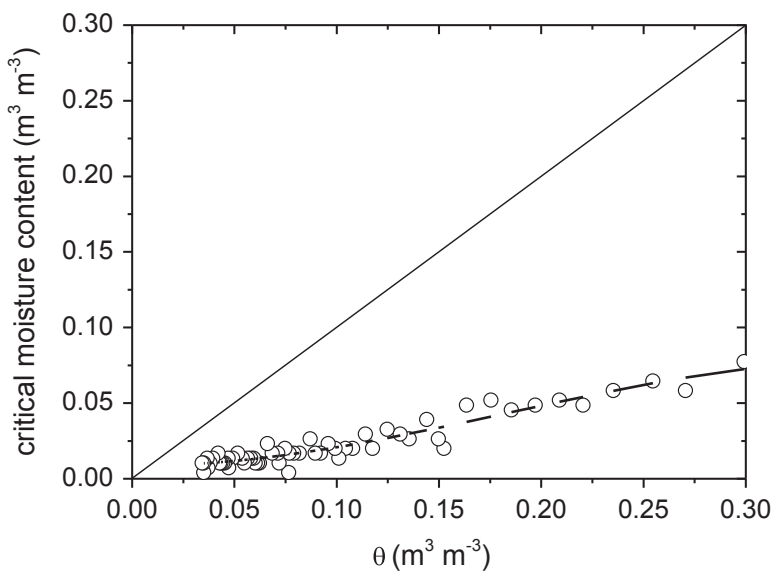

Fig. 9. Critical moisture content as a function of water content in the material for salt satured fired-clay brick with $0 \% \mathrm{RH}$ as predicted by Eq. (5).

from the concentration profiles obtained for salt solution saturated brick dried at 0\% RH (Gupta et al., 2012). Thus, most of the sample is not affected by the crystallization process. This is why we keep the same unsaturated hydraulic conductance and retention curve as for the sample without salt crystals.

Using the data shown in Fig. 4 for the case 0\% RH, Eq. (5) gives the variation of the critical moisture content shown in Fig. 9. As can be seen, the critical moisture content is never reached, i.e., $\theta$ is always greater than $\theta_{c}$ consistently with the evolution of moisture content profile given in Fig. 2.

Consistently with the experiment with a partial covering of surface with Teflon tape (see Section 3.2 and Fig. 3), the reduction of the drying rate is explained by the progressive reduction of the evaporation surface at the top of sample due to the progressive colonization of the surface by the efflorescence blocking crust. The effective evaporation surface of porous medium, which is denoted by $A_{a p p .,}$ can be computed simply from the relationship:

$A_{\text {app. }}(t)=A \frac{J(t)}{J(0)}$

where $J(0)$ is the evaporation rate at $t=0$. Using again the data shown in Fig. 4 for the case $0 \% \mathrm{RH}$, this gives the evolution of apparent evaporation surface shown in Fig. 10. $A_{a p p}$. is called the apparent evaporate surface because other factors may contribute to reducing the evaporation rate as will be briefly discussed in Section 4.4. The result shown in Fig. 10 is consistent with the progressive blocking of sample top by the development of blocking efflorescence. Using this model, the drying process can still be considered as in the constant rate period, by considering the evaporation flux at the surface free of efflorescence $j=J(t) / A_{\text {app }} .(t)$, which is constant and equal to $J(0) / A$. Thus, the mass transfer at the top surface of sample free of efflorescence is essentially the same at any time.

\subsection{Critical moisture content}

A key feature in the analysis is that the critical moisture content can be very low in a fired-clay brick as indicated by the retention curve (Fig. 7). As already mentioned, this can be due to the low percolation threshold of the pore space, which is consistent with the relatively large pore size distribution of the fired-clay brick spanning over more than three decades between a few tens of nanometers and $100 \mu \mathrm{m}$ (Pel et al., 2010). The thick liquid films that can be present along the walls and corners of pore space within the pores invaded in the bulk by the gas phase (Chauvet et al., 2009; Laurindo and Prat, 1998) may also play a role in 


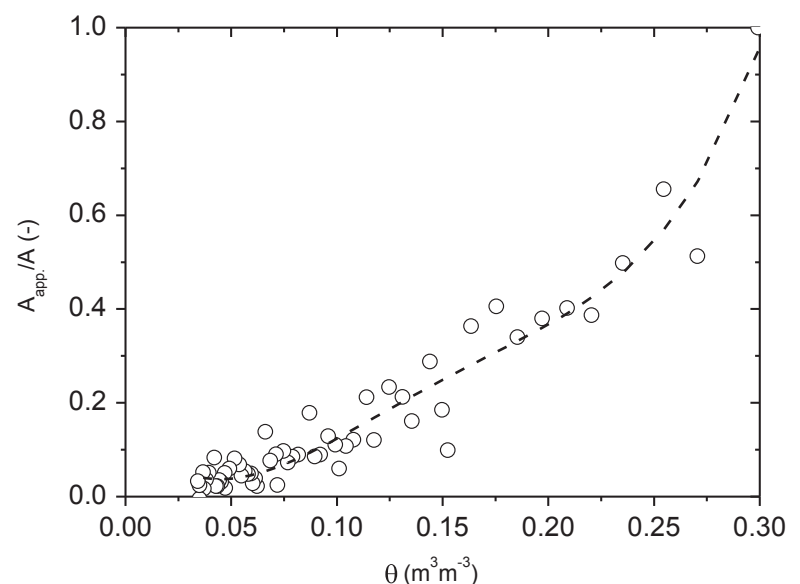

Fig. 10. Variation of apparent evaporation surface area as a function of mean moisture content as predicted by Eq. (6) for the salt solution saturated brick dried at $0 \% \mathrm{RH}$.

maintaining the hydraulic connection all over the pore space at very low moisture contents.

\subsection{Other effects}

The simple model considered in Section 4.2 essentially assumes that the system becomes a system of two layers in series as a result of crystallization: a porous layer free of crystal corresponding to almost the entirety of fired-clay brick sample connected a thin crystallization layer formed by the efflorescence crust and probably a very thin layer of the fired-clay brick sample adjacent to the top surface. The image of the crust shown in Fig. 5a suggests that the transfers are probably three-dimensional in the top region of sample since the development of salt crust is not homogeneous. Thus, there is some redirection of the flow carrying the ions underneath the porous medium surface toward the part of the surface free of efflorescence. The corresponding effects are included in the apparent surface area $A_{a p p}$. shown in Fig. 10, which thus may not exactly correspond to the evaporative surface area. It can be also noted that the development of the crust could also modify the structure of the water vapor field in the air above the sample top surface, which may slightly modify the evaporation at the surface free of efflorescence. Nevertheless, we believe that the simple model presented in Section 4.2 captures the essential of the effects explaining the moisture profiles during the drying of firedclay brick samples with and without $\mathrm{NaCl}$ as shown in Fig. 2 and the evaporation rate variations shown in Fig. 4.

\section{Conclusions}

In the case of water saturated fired-clay brick two drying stages were observed, i.e., a continous drying rate period followed by a receding drying front period. These results are in accordance with the standard drying behavior of water saturated porous media. However, at $0 \% \mathrm{RH}, \mathrm{NaCl}$ suppresses the formation of a drying front. This is due to the extremely low drying rate, which is mainly caused by pore blockage near the drying surface. The low evaporation rate at the surface maintains a continuous hydraulic connection between the surface of the porous medium and the liquid present inside the material till low saturation values. In the case of salt saturated fired-clay brick, the evaporation rate is higher at high relative humidities and salt ions crystallize as efflorescence on the surface of the brick. Because of the higher evaporation rate sufficient water transport cannot be maintained. This leads to the penetration of a front inside the brick. Sorptivity tests showed that the permeability of the brick dried at high humidities is higher than the permeability of the brick dried at low humidity due to pore blockage in the later case. Using a simple continuum scale model of drying, the critical moisture content was determined. It is shown that the critical moisture content can be very low in the case of fired-clay brick. This is due to low threshold of the pore space, which is consistent with the relatively large pore size distribution of the fired-clay brick. For salt saturated material it is shown that the critical moisture content is never reached inside the brick and is in agreement with the experimental results. Also, using the model it is shown that the critical moisture content decreases with the reduction in evaporation rate. The reduction of the drying rate is explained due to the progressive coverage of the surface due to the formation of crust like efflorescence.

Hence, drying with salt leads to a paradoxical situation in which increasing the relative humidity in the external air or reducing the external evaporation demand can increase the evaporation rate. This is explained by the dependence of efflorescence properties with potential evaporation, i.e., the evaporation rate at the very beginning of drying. Sufficiently high evaporation rates lead to the formation of blocking efflorescence (crust) whereas lower rates can lead to non-blocking efflorescence. Although some insights into the blocking-non-blocking efflorescence transition are proposed in recent works (Chauvet et al., 2009; Laurindo and Prat, 1998), this topic clearly needs further investigation so as to predict whether efflorescence will be blocking or not blocking in a given situation.

This work also indicates that the concept of critical moisture content should be considered with caution. As demonstrated here, the critical moisture content, i.e., the moisture content marking the end of stage- 1 in drying, should not be regarded as an intrinsic property of a porous material but varies depending on the evaporation condition. The lower the initial evaporation rate, the greater the critical moisture content.

\section{Acknowledgments}

We thank Hans Dalderop and Jef Noijen for their technical assistance. A part of this project is supported by the Dutch technology foundation (STW).

\section{References}

Carmeliet, J., Roels, S.J., 2002. Determination of the moisture capacity of porous building materials. J. Therm. Envel. Build. Sci. 25, 209-237.

Chauvet, F., Duru, P., Geoffroy, S., Prat, M., 2009. Three periods of drying of a single square capillary tube. Phys. Rev. Lett. 103, 124502-124505.

Eloukabi, H., Sghaier, N., Nasrallah Ben, S., Prat, M., 2013. Experimental study of the effect of sodium chloride on drying of porous media: the crusty-patchy efflorescence transition. Int. J. Heat Mass Transf. 56, 80-93.

Espinosa-Marzal, R.M., Scherer, G.W., 2013. Impact of in-pore salt crystallization on transport properties. Environ. Earth Sci. 69, 2657-2669.

Gupta, S., 2013. Sodium Chloride Crystallization in Drying Porous Media: Influence of Inhibitor (Ph.D. thesis), The Netherlands.

Gupta, S., Terheiden, K., Pel, L., Sawdy, A., 2012. Influence of ferrocyanide inhibitors on the transport and crystallization processes of sodium chloride in porous building materials. Cryst. Growth Des. 12 (8), 3888-3898.

Hall, C., Hoff, W.D., 2002. Water Transport in Brick, Stone and Concrete. Spon Press, London, pp. 188-194

Huinink, H.P., Pel, L., Michels, M.A.J., 2002. How ions distribute in a drying porous medium - a simple model. Phys. Fluids 14, 1389-1395.

Kopinga, K.. Pel, L., 1994. One dimensional scanning of moisture in porous materials with NMR. Rev. Sci. Instrum. 65, 3673-3681.

Laurindo, J.M., Prat, M., 1998. Numerical and experimental network study of evaporation in capillary porous media. Drying rates. Chem. Eng. Sci. 53, 2257-2269.

Le Bray, Y., Prat, M., 1999. Three-dimensional pore network simulation of drying in capillary porous media. Int. J. Heat Mass Transf. 42, 4207-4224.

Lewin, S.Z., 1982. Conservation of Historic Stone Buildings and Monuments. National Academy Press, Washington D.C., pp. 120-144 
Mualem, Y., 1976. A new model for predicting the hydraulic conductivity of unsaturated porous media. Water Resour. Res. 12, 513-522.

Mujumdar, A.S., 1995. Handbook of Industrial Drying, 2nd ed. Marcel Dekker, New York

Nachshon, U., Weisbrod, N., Dragila, M.I., Grader, A., 2011. Combined evaporation and salt precipitation in homogeneous and heterogeneous porous media. Water Resour. Res. 47, W03513.

Pel, L., Sawdy, A., Voronina, V., 2010. Physical principles and efficiency of salt extraction by poulticing. J. Cult. Herit. 11, 59-67.

Peysson, Y., Bazin, B., Magnier, C., Kohler, E., Youssef, S., 2011. Permeability alteration due to salt precipitation driven by drying in the context of $\mathrm{CO}_{2}$ injection. Energy Proc. 4, 4387-4394.

Prat, M., Bouleux, F., 1999. Drying of capillary porous media with a stabilized front in two dimensions. Phys. Rev. E 60, 5647-5656.
Prat, M., 2007. On the influence of pore shape, contact angle and film flows on drying of capillary porous media. Int. J. Heat Mass Transf. 50, 1455-1468.

Scherer, G.W., 1999. Crystallization in pores. Cem. Concr. Res. 29, 1347-1358.

Taber, S., 1916. The growth of crystals under external pressure. Am. J. Sci. 41, 532-556.

Van Genuchten, M.T., 1980. A closed-form equation for predicting the hydraulic conductivity of unsaturated soils. J. Am. Soc. Soil Sci. 44, 892-898.

Veran-Tissoires, S., Marcoux, M., Prat, M., 2012a. Salt crystallization at the surface of a heterogeneous porous medium. Europhys. Lett. 98 (34005), p1-p6.

Veran-Tissoires, S., Marcoux, M., Prat, M., 2012b. Discrete salt crystallization at the surface of a porous medium. Phys. Rev. Lett. 108, 054502-054505. 